\title{
2020
}

Author information:

Louie G. Simbajon

lgsimbajon.personal@gmail.com orcid: 0000-0003-0359-8610

Gia Lorraine C. Mata gialorrainematal6@gmail.com orcid no: 0000-0003-2413-7143

Dessa Grace L. Ybañez dgybanez.usep@gmail.com orcid no: 0000-0001-7050-3189

Bachelor of Science in Information

Technology (2019)

College of Teacher Education and Technology

University of Southeastern Philippines

Tagum-Mabini Campus,

Apokon, Davao del Norte

Dhally A. Ilisan

dhally.ilisan@usep.edu.ph orcid no: 0000-0003-1217-2099

Faculty Department of Information

Technology

College of Teacher Education

and Technology

University of Southeastern Philippines

Tagum-Mabini Campus,

Apokon, Davao del Norte

\section{DigiMango: A Digital Postharvest Evaluation Tool on Mango Fruit}

\author{
Louie G. Simbajon, Gia Lorraine C. Mata, \\ Dessa Grace L. Ybañez, and Dhally A. Ilisan
}

\section{$\underline{\text { Abstract }}$}

Mango is one of the most important fruits in the Philippines, with the Carabao variety being the most widely grown dominating the export market. However, the production of Carabao mangoes for export has dropped due to insect pests and diseases, resulting in serious postharvest decay. Anthracnose is the most common of these diseases. DigiMango is a mobile application that identifies the severity of anthracnose in Carabao mangoes during postharvest. The application offers to quantitatively evaluate the development of spot-like lesions on the surface of the mango and produce disease ratings. However, because this application is created for use in laboratory conditions, the mangoes used in the planning and testing were already pre-evaluated and the diseases pre-identified, with the application's sole purpose providing convenience and objective rating evaluations to the researchers. The application was able to map the lesions by capturing the four sides of the mango using the smartphone's camera and converted the process using a combination of the following: canny edge detection, morphological transformation, contour approximation, and a thresholding algorithm. After thorough evaluation of the application, the study has proven that the use of the DigiMango application is acceptable and positively correlated with the experts' rating. Any difference with the hedonic scales used by the researchers is not statistically significant.

Keywords: postharvest diseases, image processing, anthracnose severity, mango disease 
Mango (Mangifera indica) is a tree of the Anacardiacease family, whose fruit has been consumed for thousands of years (Yadav, Yadav, \& Singh, 2018), and is an emerging tropical export crop produced in about 90 countries in the world, with a production of over 25.1 million tons (Chauhan, Gupta, \& Agrawal, 2014).

The Carabao variety, produced by the Philippines, is currently the sole variety dominating the export market (Office of the USec, 2018). In 2015, the country ranked seventh among fresh and dried mango exporters worldwide, with US\$91 million in profits and a 4\% share of the global market (FernandezStark, Couto, \& Gereffi, 2017). However, a Department of Agriculture study found that Carabao mango production declined sharply in 2017 by as much as 19\% (Office of the USec, 2018). Factors contributing to this decline in mango production include damage from insect pests and diseases, the practice of excessive flower induction among older trees, and the failure to adopt appropriate cultural practices, like fertilization, pruning, and fruit bagging.

Onyeani \& Amusa (2015) observed that anthracnose is a most devastating fungal disease, limiting the production and marketing of fresh mango fruits in Southwest Nigeria. The study also investigated anthracnose incidence and severity in mango, and found that Colletotrichum gloesporiodes, a fungus that causes anthracnose, was particularly prevalent during postharvest.

Anthracnose is the most serious fungal disease affecting mangoes in the Philippines, impacting Carabao mango exports by ripening them faster and dropping their price competitiveness in the world market. With the alarming problem encountered by mango growers, the Department of Agriculture coordinated with state universities and colleges (SUCs) and experts to conduct a research on Carabao mango diseases (Office of the USec, 2018), but the practice in evaluating anthracnose disease was based on visual 'hedonic' scales (Wichchukit \& O'Mahony, 2015), a qualitative approach that depends on the experience of the evaluators. This practice is currently the standard scaling used worldwide, where mango experts evaluate the fruits individually to analyze the disease severity during the postharvest phase. The data gathered through this evaluation was then used for the further study of appropriate medicines and fertilizers to control anthracnose.

This makes a digital postharvest evaluation tool on mangoes critical in assisting mango researchers and experts in monitoring the development of anthracnose in Carabao fruits during postharvest.

DigiMango is an offline application for the Android OS used for capturing, processing, and analyzing digital images to help quantitatively evaluate a mango's anthracnose severity using the smartphone's built-in camera. It aims to evaluate the percentage of anthracnose lesions in individual fruits per transaction, calculate anthracnose disease rating in mango fruits at a maximum 
of ten samples per transaction, and save and store the past ratings of transactions for future reference along with additional information.

The application captures four (4) sides of the mango fruit and measures the percentage of the total surface area where the disease is visible. The application includes a presentation of the scales and the disease's severity rating with the added sample evaluation, as well as options to either save the result for future use or delete when found unnecessary.

An option to have bulk and individual mango evaluation is available, with a maximum of ten (10) mangoes for bulk entries, and fruits captured in bulk entries can be rated individually. The application considers illumination for desirable results, meaning it must only be used indoor and the user should turn on the phone's flash. The examined fruits must be placed on a white coloured background, such as a white cartolina, to avoid color miscalculations, and the background's size must be twenty-two (22) by twenty-eight (28) inches (cartolina size). When taking pictures of the samples, the frame must be from one edge of the background to the other edge; this is advised to further address the differences of the focal length of the smartphone cameras.

DigiMango is a tool used to quantitatively evaluate the development of spotlike lesions on the surface of mango fruit, and assess this (from mild infection to severe infection) based on the percentages of the affected areas in the sampled fruits: $0 \%-5.9 \%$ (no infection), $6 \%-55.9 \%$ (average infection), and 56\%$100 \%$ (severe infection) depending on the scope of the area affected by the anthracnose lesion. Every transaction is considered as one project, with each project allowing a minimum of one (1) and a maximum of ten (10) mangoes to be captured at the same time. The application shows the results of individual ratings and the evaluation of anthracnose lesions per sample. Additionally, the user can add more mangoes in one project. After the result of one transaction is shown, an option is presented where the user can capture another set of mangoes. As a result, both the first and the latest transactions would be presented as one, and the user can either save or delete the said results.

DigiMango is currently limited to the quantitative assessment of disease severity on fruit surfaces, and cannot further diagnose the disease, as the mobile application is made for mango researchers/experts/laboratory technicians. Furthermore, the application does not include any instructions on how to treat the said disease, and is only limited to mango evaluation in agricultural purposes. Other fruits or diseases cannot be validated as needed.

With this application, the researchers aim to help mango disease researchers and plant technicians in postharvest fertilizer product experimentations. This would provide data necessary for the enhancement of postharvest processes, and serve as the basis for future actions to improve fruit quality in subsequent yields. 


\section{Materials and Methods}

This work includes a flow chart to ensure that the system's process is properly organized. The images of the fruit/s were first acquired using a high resolution camera for better results and efficiency. Image processing techniques were then applied to the images to extract features required for further analysis.

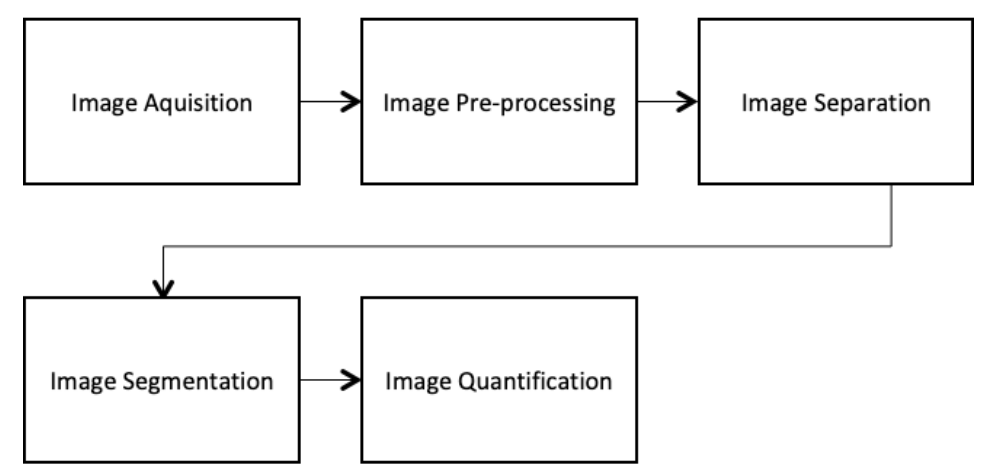

Figure 1. Block Diagram of Application's Image Processing Technique

Figure 1 shows a basic block diagram of the application's process. Each step is further explained below:

\section{Image Acquisition}

Four images of a single fruit, or a set of fruits with four (4) sides, were acquired using a camera. The fruits were placed on a white background with minimal light reflection for better results. The camera should be held horizontally to the plane of the fruit, with the distance adjusted in a way that the photograph shows only the background and the fruit itself. For bulk fruit acquisitions, an approximate distance of 1 inch from one fruit to another should be strictly observed.

In evaluating the percentage of anthracnose lesions in a mango fruit, pictures of the four sides of a mango fruit are needed, as shown in Figure 2 (a). These are the $\mathrm{A}$ and $\mathrm{B}$ sides that correspond to the front and back parts of the mango, and the $\mathrm{C}$ and $\mathrm{D}$ sides that correspond to the left and right parts of the mango. After acquiring the images, the user can start the process by tapping the "start process" button. The application then evaluates and displays the percentage of anthracnose lesion found in four sides of the mango fruit, along with its degree of infection, as shown in Figure 2(b). 


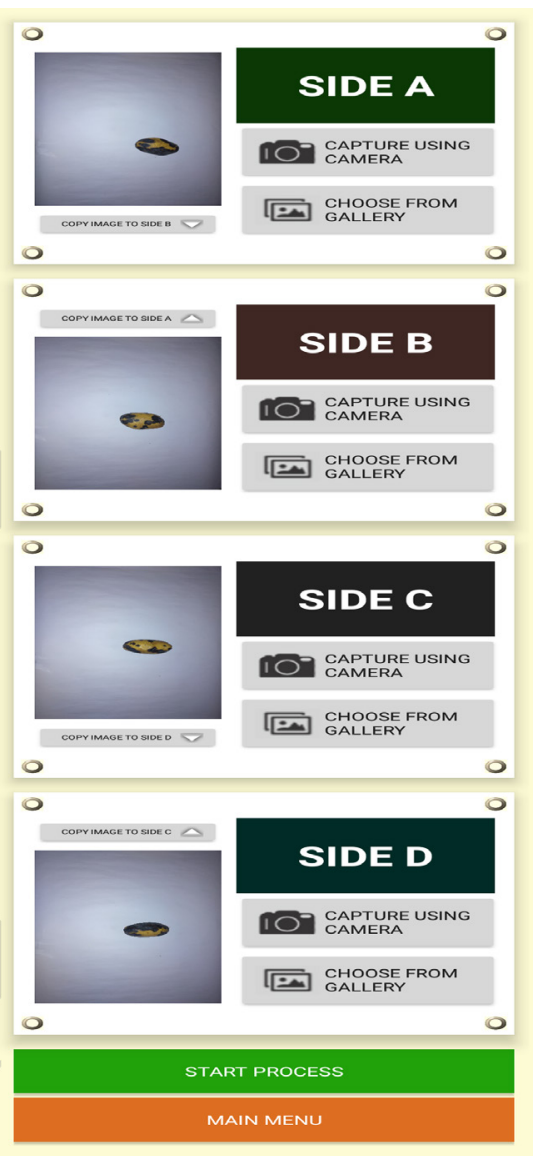

(a)

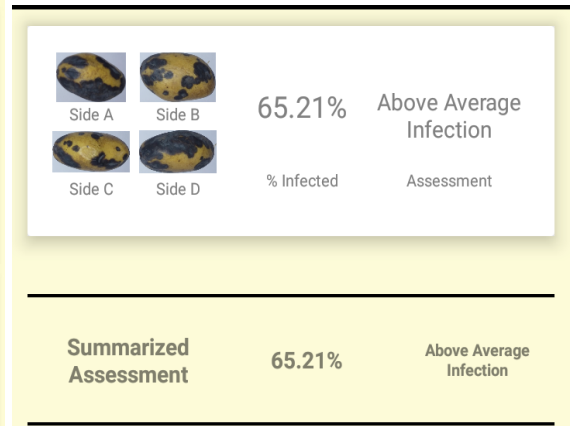

\section{Research Name}

\section{Area/Source}

\section{Tree Size}

\section{Treatment}

\section{Replicate}

Figure 2. (a) Image acquisition of the application; (b) Evaluation result of the processed image. 
Figure 3 shows a sample photo of ten mango fruits that were calculated and evaluated individually. During the individual rating, each side of a mango was captured and paired to respective individual cropped photos. To get precise results, the mangoes must be at least one inch apart from each other to allow for the mangoes to be cropped accordingly. Since ten mangoes would not fit in one row given the background's allowable size, the fruits were arranged in two rows with the same distance from each other. It should be noted that in the process of capturing the four sides of the mango, the mango must not be interchanged for it significantly affects the pairing and results of the rating.

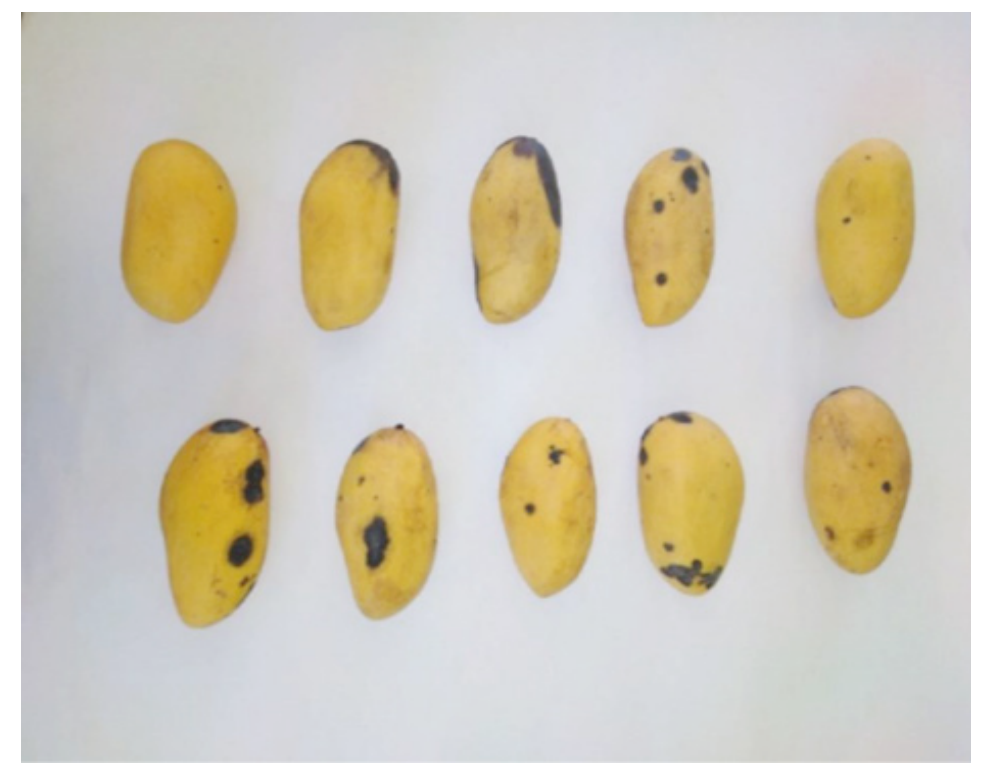

Figure 3. Position of ten (10) mangoes when captured

\section{Image pre-processing}

The captured images undergo pre-processing. In this approach, the captured images are processed using canny edge detection to detect the mango's features, with a lower-upper threshold ratio of 20:250 to detect a wide range of edges. Then, elements of the image are structured by passing the kernel size and shape in order to get the desired kernel and the rectangular structure of every mango in the image. 


\section{Image separation}

The pre-processed images undergo image separation to examine each mango fruit individually, with each image initially separated by performing Morphological Transformation through Dilation, followed by Erosion (useful in closing small holes inside foreground objects in the image). The Contour Approximation Method is then used to find boundaries of shapes with similar intensity while also removing all redundant points and compressing the contour, which saves memory space. The contour area is then computed and serves as the mango's total surface area. After this, the application calculates and returns the minimal up-right bounding rectangle for the specified point set to crop every mango in the image.

The cropped object images is then processed for recognition using pretrained images along with TensorFlow (training the model using Python).

\section{Image segmentation}

After applying pre-processing and image separation, the outcome of the image is fed to the segmentation stage. Segmentation is carried out based on histogram thresholding and morphological operations (Sudharani, et al., 2016). Here, color space segmentation is used by converting image into HSV color space.

The image is processed by segmenting the color between the mango and the background before collecting the value of the mango, in order to ensure that background colors do not interfere in image quantification. Images are converted from a BGR to a YUV color encoding system, with the "Y" channel extracted to perform thresholding. The thresholding is then implemented with a minimum value of 100 and a maximum value of 255 using the following algorithms:

Inverted Binary Thresholding. According to Bangare \& Patil (2013), transforming a grayscale image to a binary image is based on the formula:

$$
\operatorname{dst}(x, y)= \begin{cases}0 & \text { if } \operatorname{src}(x, y)>\text { thresh } \\ \operatorname{maxval} & \text { otherwise }\end{cases}
$$


For every pixel, the same threshold value is applied. If the pixel value is smaller than the threshold, it is set to 0 . Otherwise, it is set to a maximum value. The dst $(\mathrm{x}, \mathrm{y})$ is the destination image, which stores the result of thresholding. The $\operatorname{src}(x, y)$ is the source image, which should be a grayscale image. The value 0 is the threshold value which is used to classify the pixel values. Maxval is the maximum value which is assigned to pixel values exceeding the threshold.

Otsu's Binarization. This determines an optimal global threshold value from the image histogram. After applying Inverted Binary Thresholding, the algorithm (Otsu's) then finds the optimal threshold value, which is returned as the first output.

Image quantification. The image result from segmentation is used to quantify the anthracnose disease, characterized by lesions on the fruit's surface. Image quantification starts after the values of the segmented image are collected. The nonzero pixels are calculated in the masked/processed image to determine the healthy parts of the mango. Then finally, the percentage of the healthy and infected parts of the mango is computed using the following formula:

\section{Percentage of Healthy $=[($ healthy parts / total area $) X 100]$}

where,

Percentage of Healthy is the percentage of healthy parts of the mango, while healthy parts refer to the calculated nonzero pixels in the masked/processed image to determine the mango's healthy parts. Total area is the contour area, measured in pixels, used as the total surface area of the mango.

The percentage of infected areas can also be derived from the percentage of total healthy areas in the mango,

where:

\section{Percentage of Infected $=100-$ Percentage of Healthy}

The application identifies the severity assessment scales based on the percentages of the area affected in the mango fruit from mild infection to severe infection, 
where:

the no infection is $0 \%-5.9 \%$, average infection is $6 \%-55.9 \%$, and severe infection is $56 \%-100 \%$.

\section{Results and Discussion}

\section{Evaluating anthracnose lesion percentage in a mango fruit}

The DigiMango application can evaluate and display the percentage of anthracnose lesions in a mango fruit by capturing the four (4) sides of the fruit and the level of infection. To ensure that the application results were acceptable, the researchers conducted a test between the application and an expert's evaluation using the same Carabao mango sample.

The result of the test showed a very strong correlation $(r=0.986)$ between the application and expert's rating. The t-value $=0.318$ and $\mathrm{p}$-value $=0.761$ indicate that there is no significant difference between these two samples. Moreover, the application's mean and standard deviation were 45.33 and 19.87, while the expert's were 44.56 and 21.7 .

Since the results show that application rating is consistent with expert's rating, the application rating is therefore acceptable.

\section{Calculating anthracnose in mangoes at ten (10) samples per transaction}

DigiMango is an application that depends on images. In capturing the mango fruit, considering the capturing procedures is crucial in order to produce an accurate rating of anthracnose disease severity. An option to have an evaluation of bulk and individual mango is available, with a maximum capacity of ten (10) mangoes for bulk entries. Fruits captured in bulk entries can be rated individually.

Figure 4 shows the result of calculating seven mangoes in one transaction and the average evaluation of anthracnose lesions on all mangoes. The calculation of the anthracnose disease on the mango fruit is based on the application's processing techniques, which apply image acquisition, image preprocessing, image separation, image segmentation and image quantification. 


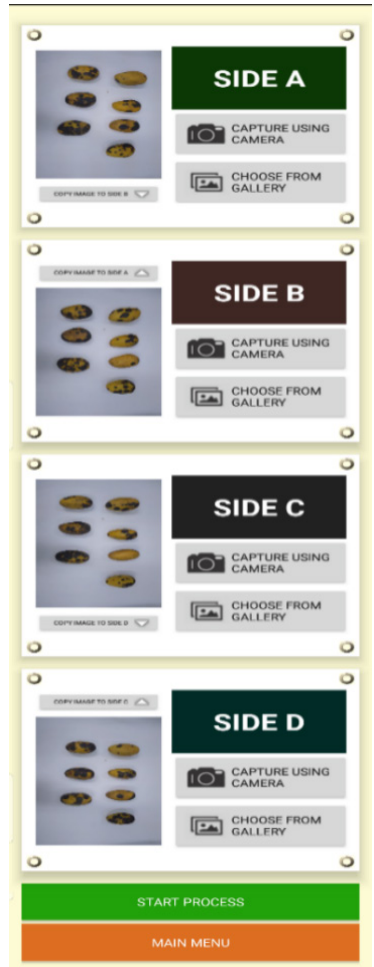

(a)

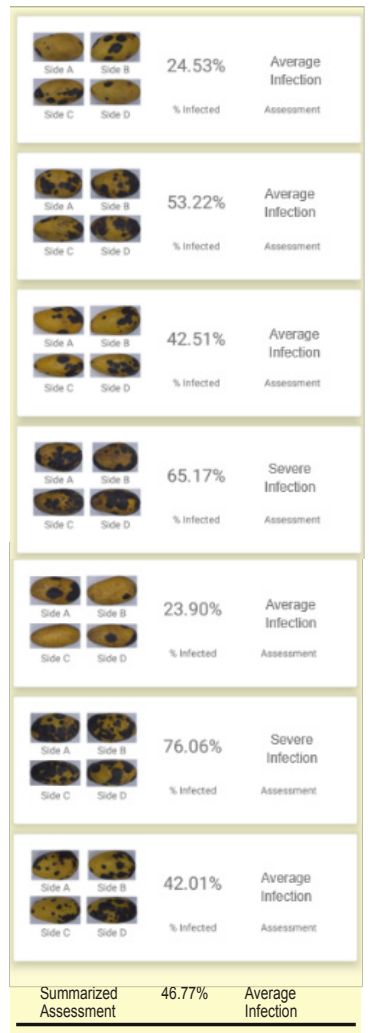

(b)

Figure 4. Evaluation of black lesion in mango fruits.

(a) Image acquisition (b) Evaluation result view

\section{Post-analysis project rating storage}

This particular functionality shows the results of user-saved transactions. Results can be saved after the evaluation display and input of details. The analyzed results display information about the samples, such as the research name, treatment used, number of replicates, source, and tree size. Figure 5 (a $\&$ b) show the archive view of the Application and the transaction result view, respectively. This is useful in storing information users need in their line of work. With this, research can be undertaken more quickly, and viewing results is easier thanks to saving options making the data readily available. 


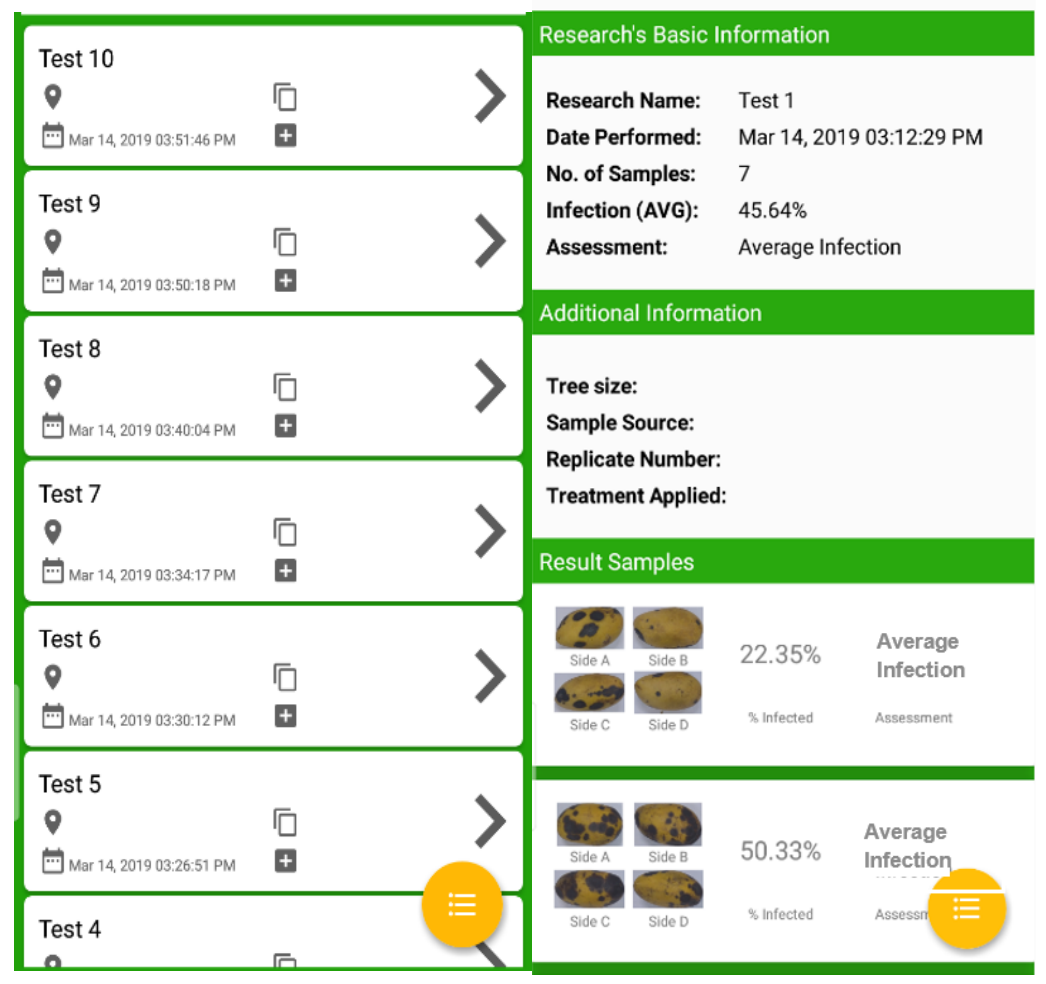

Figure 5. Screenshot of archive view: (a) past saved result, and (b) view of the saved result

\section{Recommendations}

Through the use of digital image processing, it is possible to rate anthracnose severity on mangoes. Prior to the deployment of the application, it was tested and was proven effective as it reached the $95 \%$ accuracy goal, given that all considerations were followed.

In the process of designing the application, the developers had gone through experimentation to produce accurate results. From this, certain factors were found that needed to be considered when using the application: luminance, in that the application must be used indoors with bright fluorescent lighting and that shadows can affect image processing; as well as distance between the phone and the subject because smartphone cameras have varying focusing angles such as wide and narrow focus. The developers also suggest that in capturing the 
samples, the white paper used as the background must fit within the camera frame.

Another factor was in capturing the samples' sides. The application was initially planned to capture only two, but as testing progressed, it was noted that there were parts of the mangoes that could not be calculated. This was because a mango is a three-dimensional object and the camera-generated image can only be evaluated in two dimensions. Thus, results vary.

Given this, it is advised that the application should capture all sides of the mango then do the manipulation in the code. The developers also found slight differences in the results when different brands of smartphones were used, and that different smartphones had different types of camera lenses and sensors that provided different image results despite having the same number of megapixels.

Further recommendations in order to improve DigiMango in terms of mobility and development are as follows: develop iOS and Windows compatibility, enable cloud computing backup functions to avoid data loss, and develop an additional Web-based application that can both automatically generate reports in MS Excel.

In terms of improving features, it is recommended that the application should be given a feature that will enable it to segregate lesions and spots caused by post-harvest diseases. The application should also be further developed to accommodate more than ten mangoes per process, thus reducing research time, as well as to add a postharvest disease database that will allow users to identify these various diseases and receive basic instructions in treating them. Finally, the development of online connectivity is recommended to allow users to geomap the location of mango trees and fruits affected by postharvest diseases. 


\section{References}

Bangare, S., \& Patil, S. (2015 Jan). Reviewing Otsu's Method For Image Thresholding, International Journal of Applied Engineering Research 10(9): 21777-21783.

Chauhan, S., Gupta, K. C., \& Agrawal, M. (2014). A New Approach of Hurdle technology to preserve Mango fruit with the application of Aloe vera gel and Calcium chloride. International Journal of Current Microbiology and Applied Sciences, 3(5), 926-934.

Fernandez-Stark, K., Couto, V., \& Gereffi, G. (2017 Apr). The Philippines in the Mango Global Value Chain. Retrieved from www.cggc.duke.edu

Office of the Undersecretary, High Value Crops and Rural Credit. (2018). Philippine Mango Industry Roadmap 2017-2022. Philippines: Department of Agriculture.

Onyeani, C. A., \& Amusa, N. A. (2015). Incidence and severity of anthracnose in mango fruits and its control with plant extracts in South West Nigeria. International Journal of Agricultural Research. https://doi.org/10.3923/ijar.2015.33.43

Sudharani, K., Sarma, T. C., \& Satya Prasad, K. (2016). Histogram Related Threshold Technique for Region based Automatic Brain Tumor Detection. Indian Journal of Science and Technology, 9(48). https://doi.org/10.17485/ijst/2016/v9i48/89891

Wichchukit, S., \& O’Mahony, M. (2015). The 9-point hedonic scale and hedonic ranking in food science: Some reappraisals and alternatives. Journal of the Science of Food and Agriculture, 95(11), 2167-2178. https://doi.org/10.1002/jsfa.6993

Yadav, D., Yadav, K. S., \& Singh, S. (2018). Mango: Taxonomy and Botany. Journal of Pharmacognosy and Phytochemistry, 7(2), 3253-3258. 
